\title{
Aspects of Combining Models of the Earth's Internal and External Magnetic Field
}

\author{
E. O. FLÜCKIGER and E. KOBEL \\ Physikalisches Institut, University of Bern, Sidlerstrasse 5, CH-3012 Bern, Switzerland
}

(Received September 18, 1989; Revised June 13, 1990)

\begin{abstract}
On the basis of an analysis of the 7/8 December 1982 ground-level cosmic ray event the paper specifies the limitations of any mathematical model of the main geomagnetic field in its use for cosmic ray analyses, presents procedures and problems of combining models of the Earth's internal field with models of the external geomagnetic field, and discusses the significance of the external geomagnetic field for calculations of cosmic ray particle trajectories in near-Earth space.
\end{abstract}

\section{Introduction}

Analyses of cosmic ray observations made by ground-based detectors and by satellite experiments require a detailed knowledge about the propagation of cosmic ray particles in near-Earth space. Access of cosmic ray particles to a specific point of observation is determined by the Earth's geomagnetic field and depends on the energy (rigidity) of the particles as well as on the direction of incidence. The concepts of "Cutoff Rigidities" and of "Asymptotic Cones of Acceptance" have been developed in order to deconvolve the geomagnetic field effects and to construct the cosmic ray particle spectral characteristics and anisotropy in the near-Earth interplanetary space from cosmic ray measurements on or near the Earth (LEMAITRE and VALLARTA, 1936; STÖRMER, 1955; MCCRACKEN, 1962; MCCRACKEN et al., 1968). The cutoff rigidity of a specific location on the Earth and of a specific direction of incidence is generally defined as the rigidity below which cosmic ray particles have no access to that location from the specified direction. The asymptotic (or approach) direction of a cosmic ray particle having a specific rigidity and arriving at a specific location on the Earth from a specific direction of incidence is the direction of motion which this particle had in interplanetary space prior to its interaction with the Earth's magnetic field. It is common practice nowadays to determine cosmic ray cutoff rigidities and asymptotic directions by computer simulation of particle trajectories using a mathematical model of the Earth's magnetic field (MCCRACKEN et al., 1962; SHEA and SMART, 1975). In the majority of these studies, the International Geomagnetic Reference Field (IGRF) model appropriate for a certain epoch is used to represent the Earth's magnetic field. Apart from few exceptions (e.g. GALL et al., 1984), effects of the quiescent magnetosphere are not yet generally taken into account. Although it is well known that during large geomagnetic storms the propagation of cosmic ray particles in near-Earth space is affected considerably and that significant changes in the cutoff rigidities and in the asymptotic directions occur (see e.g. DEBRUNNER and FLÚCKIGER, 1977; FLÜCKIGER et al., 1983, 1986; KUDO et al., 1987; and references therein) only a few studies have been performed on this subject utilizing 
the trajectory-tracing technique and combined models of the internal and external geomagnetic field (e.g. FLÜCKIGER et al., 1983; WILKEN et al., 1986). This proves to be a serious deficiency especially for the analysis of ground-level cosmic ray events (GLE) observed by neutron monitors at high geomagnetic latitudes and/or during time periods of enhanced geomagnetic activity (FLÜCKIGER, 1984). An example for such an event is the GLE on December 7/8, 1982. As illustrated in Fig. 1 this GLE occurred while a geomagnetic storm was in progress $(D s t \approx-80 \mathrm{nT})$. At the time of the event the cosmic ray cutoff rigidities at mid-latitudes were lowered by about $0.3 \mathrm{GV}$. For the analysis of this event, detailed quantitative information is needed about cosmic ray cutoff rigidities and asymptotic directions of cosmic ray particles for the particular perturbed geomagnetic field conditions which existed around 24:00 UT on December 7, 1982. It is impossible to explain the neutron monitor observations of this GLE without taking into account the effect of the external magnetic field. A special study has therefore been performed by calculating cosmic ray trajectories utilizing a model of the perturbed magnetospheric magnetic field (KOBEL, 1989). Based on that study the present paper outlines the general procedure and problems of combining models of the Earth's internal magnetic field with models of the external geomagnetic field and discusses the significance of the external geomagnetic field for calculations of cosmic ray particle trajectories in near-Earth space.

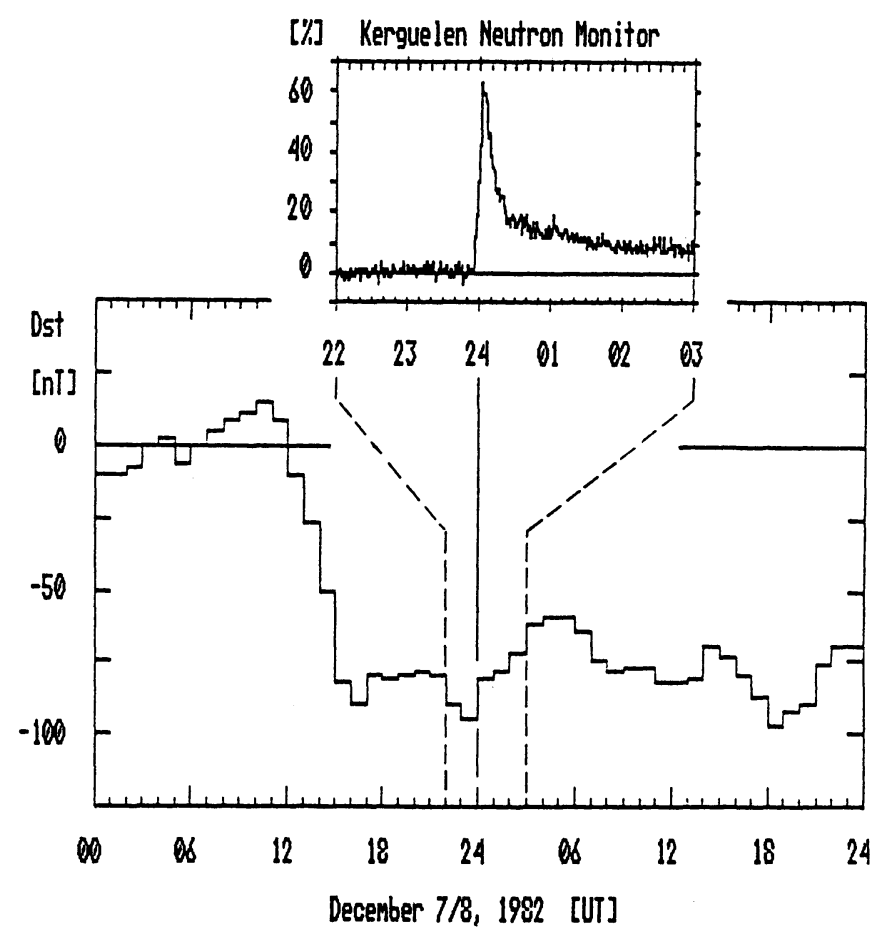

Fig. 1. The cosmic ray ground-level event on December $7 / 8,1982$. Upper panel: Relative increase in neutron monitor counting rate at Kerguelen. Lower panel: Geomagnetic index Dst. 


\section{Models of the Magnetospheric Magnetic Field}

During the past two decades, considerable progress has been made in the development of quantitative magnetospheric magnetic field models. A review of the early work including a critical comparison of different field models is found e.g. in WALKER (1979, 1983). Recently, more elaborated magnetospheric field models were presented e.g. by PFITZER et al. (1988) and TSYGANENKO (1989). For the abovementioned analysis of the 7/8 December 1982 GLE the model worked out by TSYGANENKO and USMANOV (1982) was used. Like most of the other models of the Earth's external field, this model includes the magnetic fields of the ring current, the magnetic field from the magnetotail currents as well as the magnetopause contribution and the averaged magnetic effect of fieldaligned currents. The dipole tilt angle is an input parameter, and the model refers to different levels of geomagnetic activity up to $K p \geq 3+$. An evaluation of the accuracy of the TSYGANENKO-USMANOV (1982) model has recently been given by FAIRFIELD (1988). According to this author on the dayside of the Earth the model underestimates the field strength in the low latitude region and overestimates the field strength in the cusp region. On the nightside the model underestimates the amount of field line stretching and therefore tends to underestimate the field strength off the equatorial plane. In the dawn and dusk meridian, the primary deficiency in the model is an underestimate of the tailward distortion of the field lines.

\section{Models of the Earth's Internal Magnetic Field}

In deriving magnetospheric field models from observational magnetic field data a reference model for the Earth's internal magnetic field is utilized (see e.g. MEAD and FAIRFIELD, 1975). For the TSYGANENKO-USMANOV (1982) model this reference model was the IGRF for Epoch 1965.0 (IAGA COMMISSION 2 WORKING GROUP 4, 1969). In combining models of the Earth's external magnetic field with models of the internal geomagnetic field, the corresponding reference field should in principle be used. Cosmic ray cutoff rigidities and asymptotic directions, however, are very sensitive to the secular changes in the internal geomagnetic field (SMART et al., 1987). Therefore for the study of the 7/8 December 1982 GLE the IGRF for Epoch 1980.0 was used (IAGA DIVISION 1 WORKING GROUP 1, 1981). Figure 2 illustrates the magnitude in nT of the magnetic field vector difference between the IGRF for Epoch 1980.0 and the IGRF for Epoch 1965.0. Iso-intensity contour lines are shown in the solar magnetospheric $x_{\mathrm{sm}} z_{\mathrm{sm}}$-plane for 24:00 UT on December 7, 1982. For comparison, Figure 3 shows the ratio

$$
V=\frac{\left|\boldsymbol{B}_{\text {IGRF 1980 }}-\boldsymbol{B}_{\text {IGRF 1965 }}\right|}{\left|\boldsymbol{B}_{\text {ext }}\right|}
$$

in the same representation where $\boldsymbol{B}_{\text {ext }}$ is the external field calculated according to the TSYGANENKO-USMANOV (1982) model for $K p \geq 3+$ and the same time. It can be seen that only for radial distances $\leqslant 5 \mathrm{r}_{\mathrm{e}}$ (Earth radii), where the intensity of the external magnetic field is of the order of $10 \%$ of the total magnetic field intensity or less, we find $V \geq 0$. 1 . From this and from the cosmic ray trajectory calculations discussed later we 


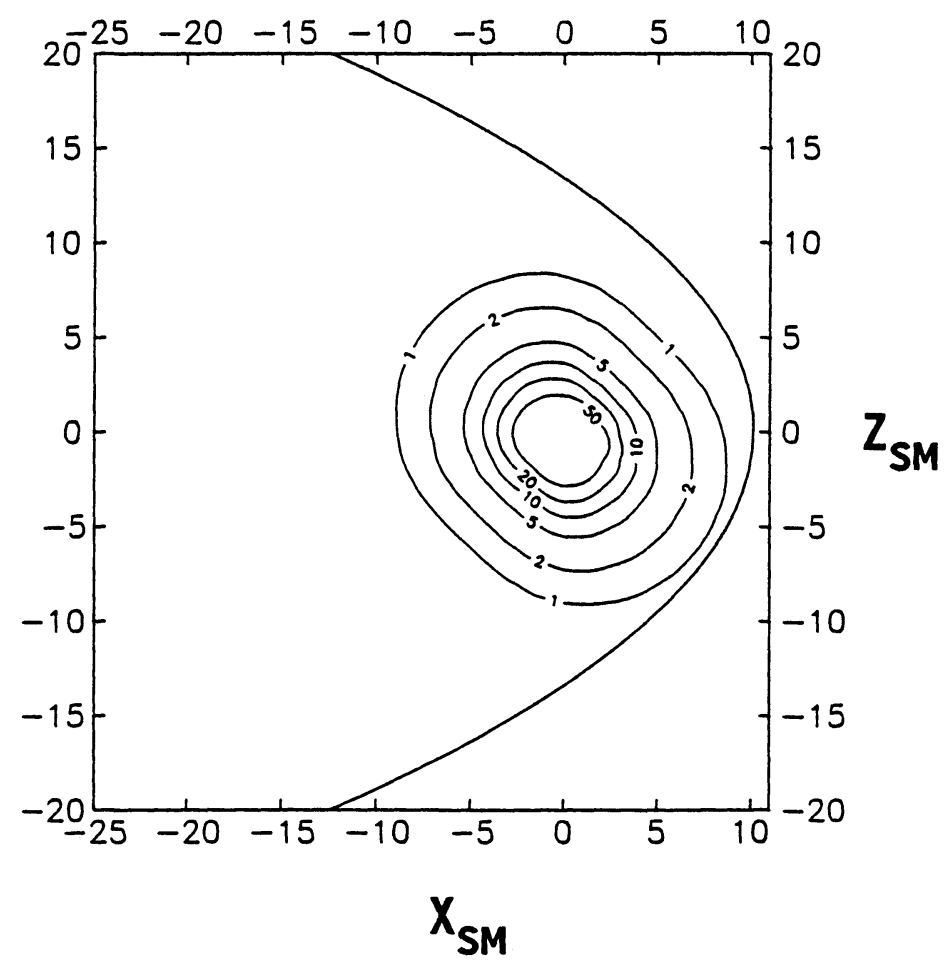

Fig. 2. Magnitude of the magnetic field vector difference between the IGRF 1980.0 and the IGRF 1965.0, as calculated for points in the solar magnetospheric $x_{\mathrm{sm}} z_{\mathrm{sm}}$-plane for December 7, 1982, 24:00 UT. Contour lines represent equal differences in $\mathrm{nT}$.

conclude that the use of the IGRF 1980.0 instead of the IGRF 1965.0 as a reference field does not lead to significant errors in the determination of the magnetospheric effects on cosmic rays.

\section{Combining Models of the Earth's External and Internal Magnetic Field}

With given models of the Earth's external and internal magnetic field the problem of combining two models into a computer code is primarily a problem of transformations between different coordinate systems. In combining the TSYGANENKO-USMANOV (1982) model with the IGRF 1980.0 the following coordinate systems are needed:

i) Geographic $(\mathrm{G})$ coordinates $(x, y, z)$ or $(r, \theta, \varphi)$ for the calculation of the geomagnetic main field, $\boldsymbol{B}^{\mathrm{M}}$;

ii) Dipole (D) coordinates $\left(x_{\mathrm{d}}, y_{\mathrm{d}}, z_{\mathrm{d}}\right)$ for the calculation of the magnetic field of the ring current, $\boldsymbol{B}^{\mathrm{R}}$;

iii) Solar magnetic $(\mathrm{MG})$ coordinates $\left(x_{\mathrm{mg}}, y_{\mathrm{mg}}, z_{\mathrm{mg}}\right)$ for the calculation of the magnetic field of the magnetopause current and of the averaged effect of field-aligned currents, $\boldsymbol{B}^{\mathrm{P}}$;

iv) Solar magnetospheric (SM) coordinates $\left(x_{\mathrm{sm}}, y_{\mathrm{sm}}, z_{\mathrm{sm}}\right)$ for the calculation of the magnetic field from the magnetotail currents, $\boldsymbol{B}^{\mathrm{T}}$. 


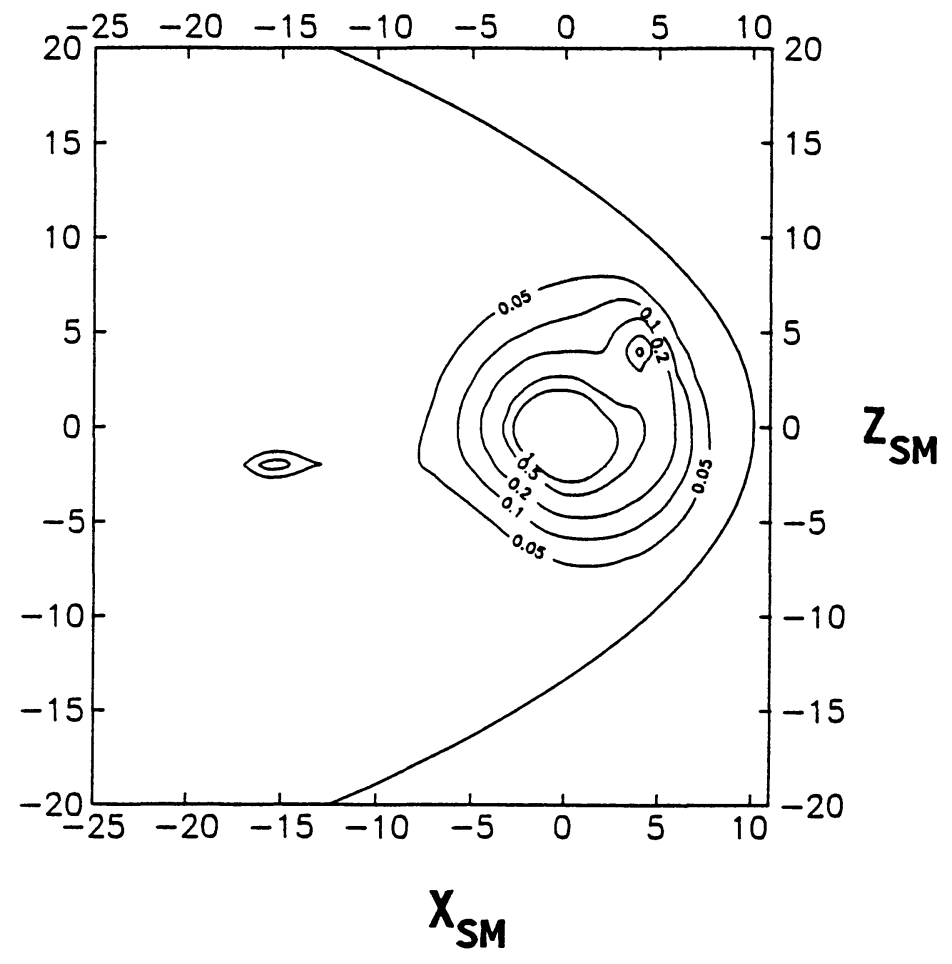

Fig. 3. Ratio between the differences in the IGRF 1980.0 and the IGRF 1965.0 as shown in Fig. 2 and the strength of the external magnetic field according to the TSYGANENKO-USMANOV $(1982)$ model $(K p \geq 3+)$ for December 7, 1982, 24:00 UT (see Eq. (1)). Contour lines represent equal ratios in the solar magnetospheric $x_{\mathrm{sm}} z_{\mathrm{sm}}$-plane.

All of these coordinate systems have their origin at the center of the Earth and each is defined by three mutually orthogonal axes. Therefore all coordinate transformations are rotations and they are made according to the usual formula

$$
\left(\begin{array}{l}
x^{\prime} \\
y^{\prime} \\
z^{\prime}
\end{array}\right)=A(t)\left(\begin{array}{l}
x \\
y \\
z
\end{array}\right) .
$$

The matrix $A$ is in general time-dependent, taking into account the Earth's motion, around its axis and around the sun. In deriving the time-dependent coefficients of the transformation matrices the algorithms published by DUFFET-SMITH (1981) were extremely useful. A detailed description of the different coordinate systems and of the corresponding transformation matrices can be found e.g. in OLSON (1970). field

For a specific location given by its geographic coordinates $(x, y, z)$ the total magnetic

$$
\boldsymbol{B}=\boldsymbol{B}^{\mathrm{M}}+\boldsymbol{B}^{\mathrm{R}}+\boldsymbol{B}^{\mathrm{P}}+\boldsymbol{B}^{\mathrm{T}}
$$


is then calculated according to the procedure shown in Fig. 4. This procedure is based on important work done by Stern (private communication). Entry 1 is used for the first call of the program whereas entry 2 refers to all subsequent calls. Several subprograms are used to evaluate the different terms of Eq. (3). The function of each subprogram is explained in Fig. 5.

No special programming tricks were used to optimize the economic feasibility of the computations. For the IGRF 1980.0 a computer code based on the standard representation of the geomagnetic main field by spherical harmonics, as adapted by STERN (1976), was utilized. The contributions of the external magnetic field were evaluated according to the mathematical expressions and parameters given by TSYGANENKO and USMANOV

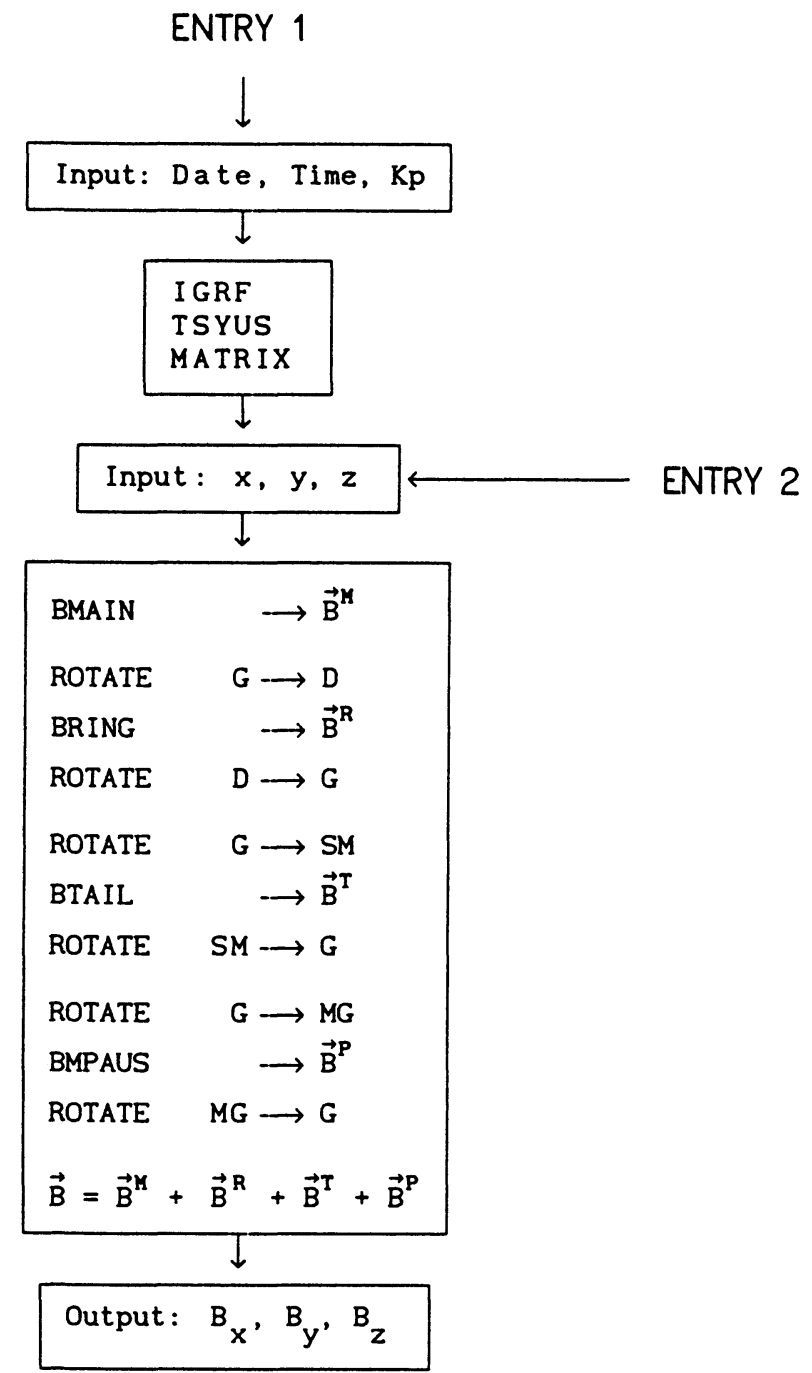

Fig. 4. Flow chart for the calculation of the combined internal and external magnetic field. For details see text. 


\begin{tabular}{|c|c|}
\hline Name & Function \\
\hline IGRF & $\begin{array}{l}\text { reads coefficients of IGRF and determines necessary } \\
\text { constants }\end{array}$ \\
\hline TSYUS & $\begin{array}{l}\text { reads parameters of the magnetospheric field model } \\
\text { according to Tsyganenko and Usmanov (1982) }\end{array}$ \\
\hline MATRIX & $\begin{array}{l}\text { generates matrices for coordinate transformations } \\
\text { (depending on date and time) }\end{array}$ \\
\hline BMAIN & $\begin{array}{l}\text { determines the geomagnetic main field, } \vec{B}^{M} \text {, in geo- } \\
\text { graphic coordinates }\end{array}$ \\
\hline ROTATE & performs necessary coordinate transformations \\
\hline BRING & $\begin{array}{l}\text { determines the magnetic field of the ring current, } \\
\vec{B}^{R} \text {, in dipole coordinates }\end{array}$ \\
\hline BMPAUS & $\begin{array}{l}\text { determines the magnetic field of the magnetopause } \\
\text { currents, } \vec{B}^{P} \text {, in solar magnetic coordinates }\end{array}$ \\
\hline BTAIL & $\begin{array}{l}\text { determines the magnetic field from the magnetotail } \\
\text { currents, } \vec{B}^{T} \text {, in solar magnetospheric coordinates }\end{array}$ \\
\hline
\end{tabular}

Fig. 5. Functions of the different subprograms shown in Fig. 4.

(1982). Under these circumstances calculations of the geomagnetic field including both the internal and external sources are only by $40 \%$ at most more time consuming than calculations using the internal sources alone.

5. The Magnetopause

For cosmic ray studies relating observations at a specific location with particle fluxes outside the domain of the Earth's magnetic field the points of entry of cosmic ray particles into the Earth's magnetosphere and the directions of their velocity vector prior to the entry into the magnetosphere (direction of approach, asymptotic direction) are of 
importance (GALL et al., 1984). Only a few of the published magnetospheric field models include a mathematical representation of the magnetopause (e.g. TSYGANENKO, 1976). For the TSYgANENKO-USMANOV (1982) model and the time around 24:00 UT on December 7, 1982 a suitable representation of the magnetopause was found to be a paraboloid with rotational symmetry around the $x_{\mathrm{sm}}$-axis, defined by

$$
x_{\mathrm{sm}}=a\left(y_{\mathrm{sm}}^{2}+z_{\mathrm{sm}}^{2}\right)+b
$$

with $a=-0.05638 \mathrm{r}_{\mathrm{e}}^{-1}$ and $b=10.11 \mathrm{r}_{\mathrm{e}}$. The parameters $a$ and $b$ were determined in such a way that the resulting magnetopause represents a smooth envelope to the magnetic field lines in the $x_{\mathrm{sm}} z_{\mathrm{sm}}$-plane as well as of the crossing points of the field lines with the $x_{\mathrm{sm}} y_{\mathrm{sm}}$-plane. Figure 6 shows the shape of this magnetopause in the $x_{\mathrm{sm}} z_{\mathrm{sm}}$-plane, together with a projection onto this plane of the magnetic field lines originating from the surface of the Earth at $y_{\mathrm{sm}}=0$, calculated again for 24:00 UT on December 7, 1982. Figure 7 shows a similar plot illustrating the total magnetic field intensity at $y_{\mathrm{sm}}=0$ for the same time.

6. Implementation of the Magnetic Field Models in a Cosmic Ray Trajectory Program

Once a computer code has been worked out which is able to evaluate the components of the total magnetic field according to Eq. (3) at any point given by its coordinates, and with a suitable mathematical representation of the magnetopause, the

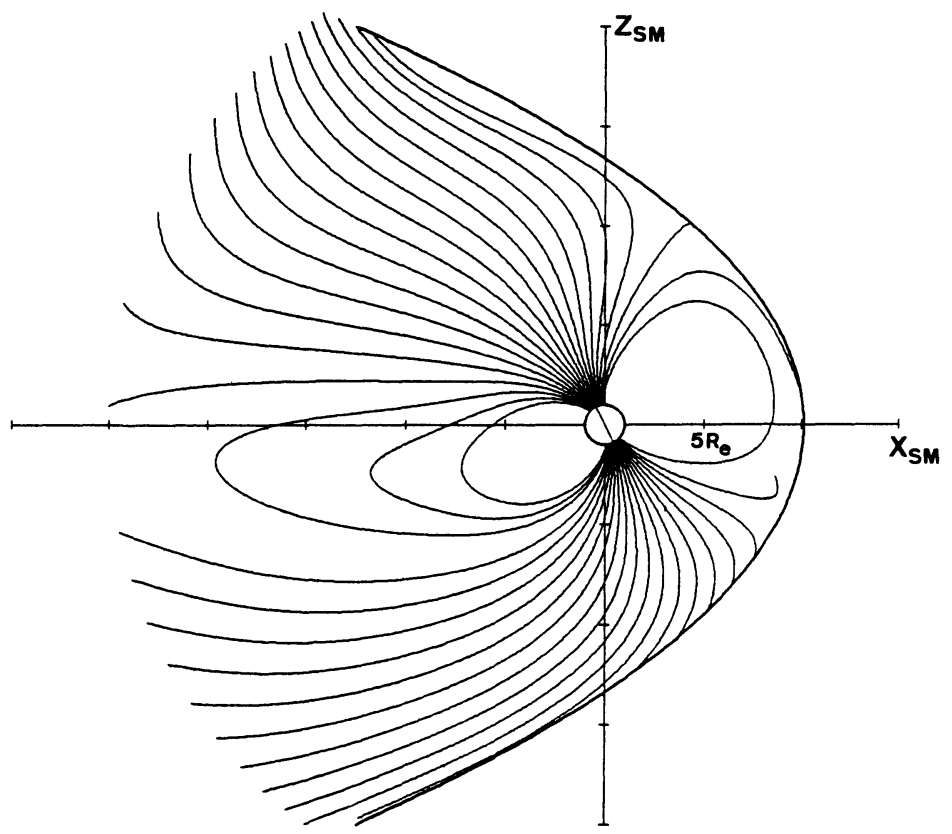

Fig. 6. Shape of the magnetopause in the $x_{\mathrm{sm}} z_{\mathrm{sm}}$-plane and projection onto this plane of magnetic field lines originating at the Earth's surface at $y_{\mathrm{sm}}=0$. For details see text. 


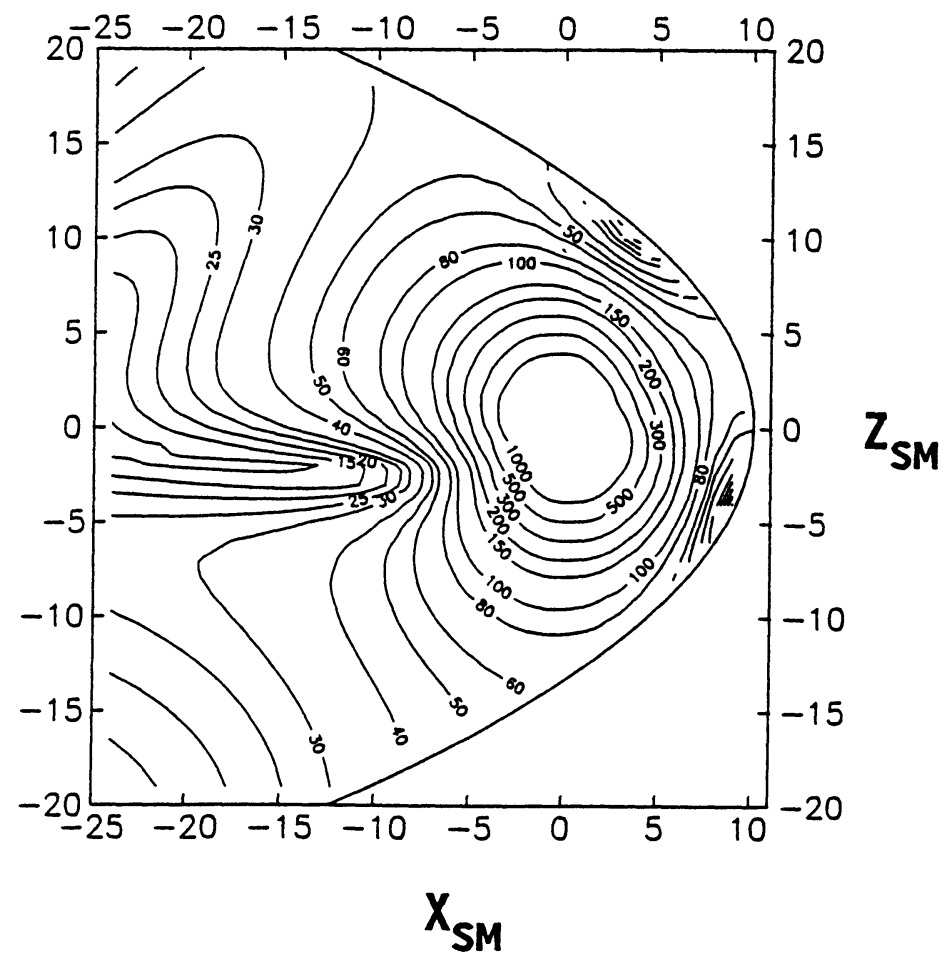

Fig. 7. Strength of the total magnetic field in the $x_{\mathrm{sm}} z_{\mathrm{sm}}$-plane on December 7, 1982, 24:00 UT according to the combined models of the Earth's internal and external magnetic field as described in the text.

implementation of the combined external and internal magnetic field models into a cosmic ray trajectory-tracing program is straightforward. A possible procedure is illustrated in Fig. 8. In accordance with the output of the magnetic field calculations shown in Fig. 4, the numerical integration of the differential equation describing the path of a particle is performed in geographical spherical coordinates. We utilized the standard method of tracing cosmic ray trajectories through the geomagnetic field, i.e. the GILL (1951) modification of the Runge-Kutta iteration procedure, as adapted by MCCRACKEN (1962), MCCRACKEN et al. (1962), and SHEA and SMART (1975).

\section{Significance of the Earth's External Magnetic Field for Cosmic Ray Cutoff Rigidities and Asymptotic Directions}

Utilizing the procedure presented in this paper, cosmic ray cutoff rigidities and directions of approach referring to December 7, 1982, 24:00 UT, have been calculated for 40 stations of the worldwide network of neutron monitors. Table 1 illustrates the results obtained for the cutoff rigidities. The cutoff rigidities were decreased worldwide. At higher latitude locations the amplitude of the decrease in the effective vertical cutoff rigidity was typically $0.2-0.3 \mathrm{GV}$ with respect to the values referring to the quiescent (internal) geomagnetic field conditions. Figure 9 demonstrates a characteristic example of the results obtained for the directions of approach (asymptotic directions). In this plot 


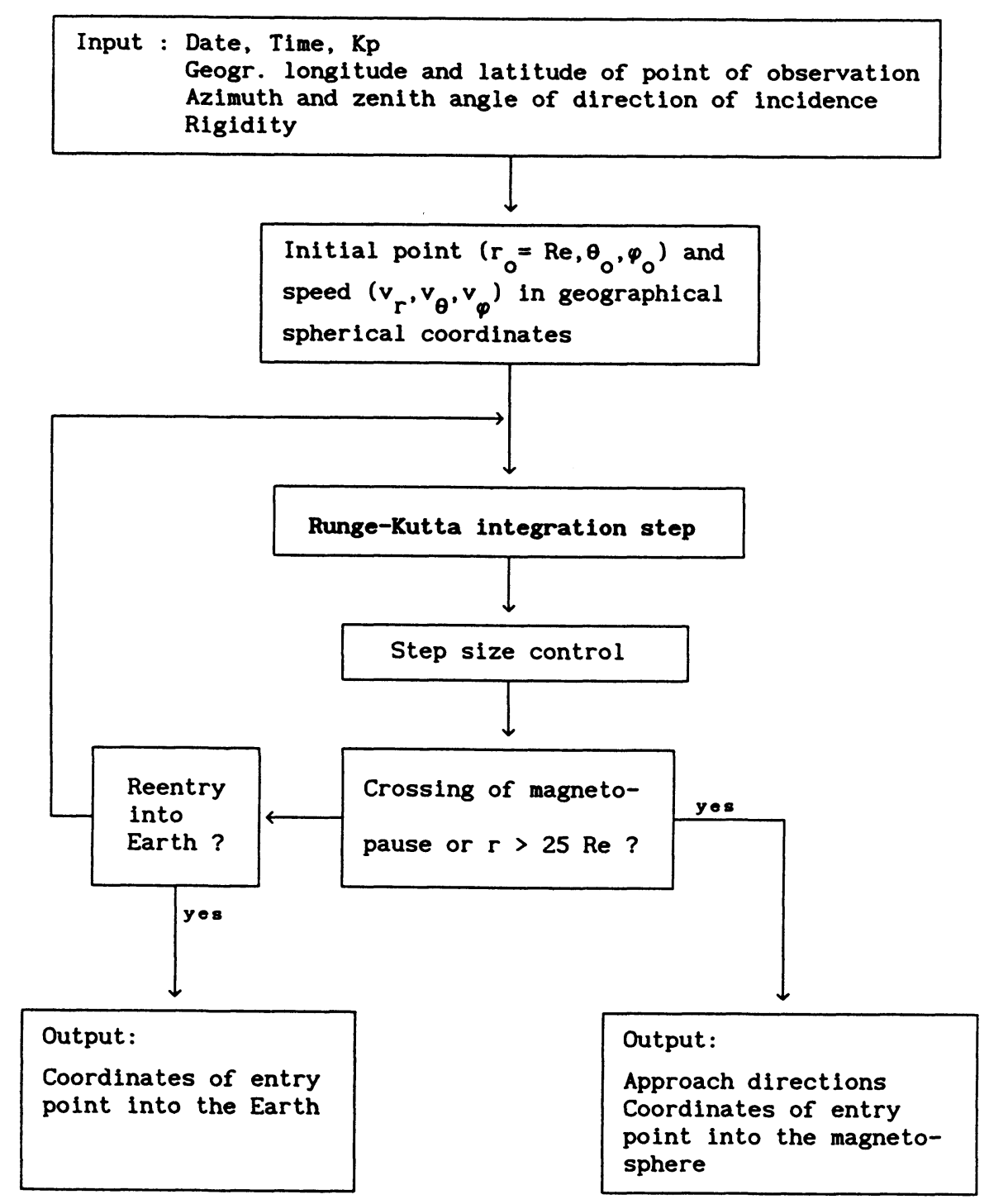

Fig. 8. Flow chart of the cosmic ray particle trajectory-tracing program.

we show the directions of approach in geographic coordinates for cosmic ray particles arriving from the vertical direction at Apatity (geographic latitude: $67.55^{\circ}$, geographic longitude: $\left.33.33^{\circ}\right)$. It can be seen that for rigidities below $10 \mathrm{GV}$ the changes are significant. The asymptotic directions are shifted both in longitude and latitude. For particles of $1 \mathrm{GV}$ the shift is of the order of $45^{\circ}$ to the east and of about $15^{\circ}$ to the north.

8. Problems and Limitations

The TSYganenko-Usmanov (1982) model is restricted to geocentric distances 
Table 1. Effective vertical cutoff rigidities as calculated for 13 selected neutron monitor locations with the combined TsYganenKo-Usmanov (1982) $(K p \geq 3+)$ and IGRF 1980.0 models for December 7, 1982, 24:00 UT, compared to the values obtained using the IGRF 1980.0 alone.

\begin{tabular}{lccc}
\hline Station & $\begin{array}{c}\text { December 7, 1982 } \\
24: 00 \mathrm{UT} \\
(\mathrm{GV})\end{array}$ & $\begin{array}{c}\text { IGRF 1980 } \\
(\mathrm{GV})\end{array}$ & $\begin{array}{c}\Delta \boldsymbol{R}_{\mathrm{c}} \\
(\mathrm{GV})\end{array}$ \\
\hline Apatity & 0.28 & 0.60 & -0.32 \\
Deep River & 0.90 & 1.11 & -0.21 \\
Goose Bay & 0.31 & 0.63 & -0.32 \\
Inuvik & 0.06 & 0.17 & -0.11 \\
Jungfraujoch & 4.49 & 4.59 & -0.10 \\
Kerguelen & 0.91 & 1.12 & -0.21 \\
Kiel & 2.22 & 2.39 & -0.17 \\
Magadan & 1.95 & 2.11 & -0.16 \\
Mawson & 0.00 & 0.20 & -0.20 \\
Oulu & 0.50 & 0.79 & -0.29 \\
Sanae & 0.58 & 0.88 & -0.30 \\
South Pole & 0.00 & 0.09 & -0.09 \\
Tixie Bay & 0.25 & 0.46 & -0.21 \\
\hline
\end{tabular}

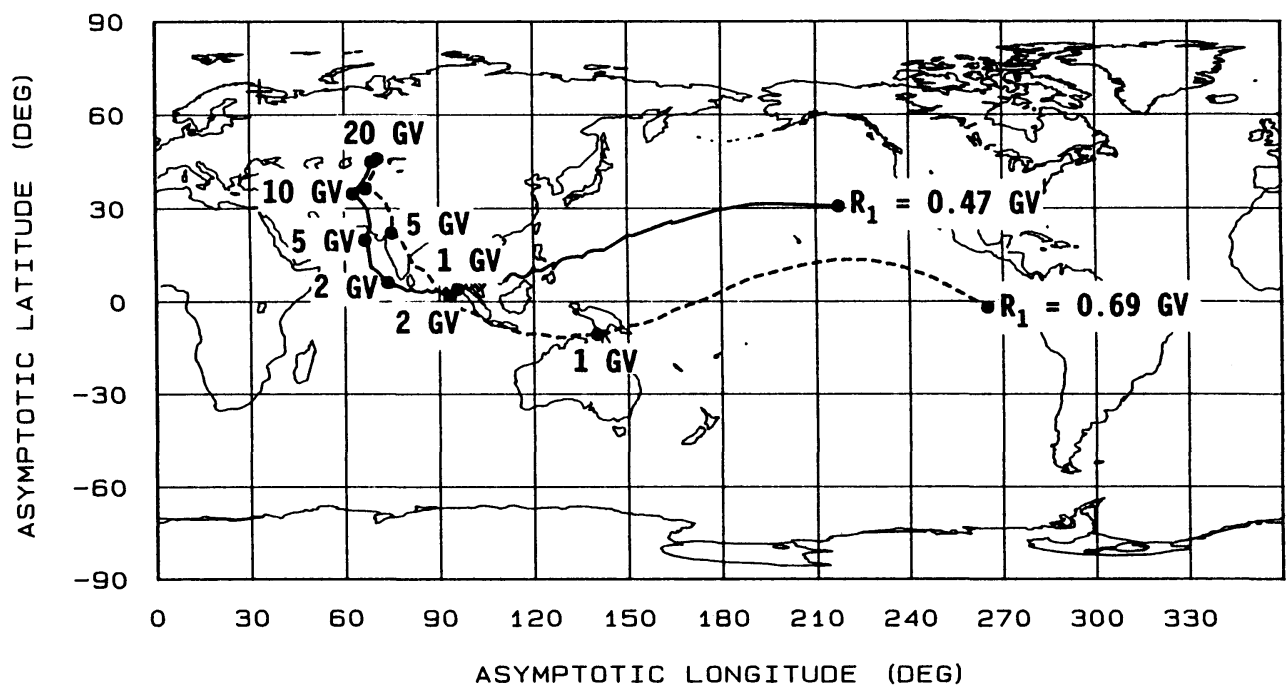

Fig. 9. Directions of approach (asymptotic directions) in geographic coordinates for cosmic ray particles arriving from the vertical direction at Apatity. Full line: December 7, 1982, 24:00 UT, combined models of the Earth's internal and external field. Dashed line: IGRF 1980.0.

smaller than $25 \mathrm{r}_{\mathrm{e}}$. As a consequence the entry points of the cosmic ray particles into the Earth's magnetosphere had to be defined either as the crossing points of the particle trajectories with the magnetopause or, in the tail region, with a sphere of radius $25 r_{e}$. In order to estimate the uncertainty introduced by this deficiency, we crudely modified the magnetospheric field model by extending the magnetic field at $25 \mathrm{r}_{\mathrm{e}}$ to the tailward direction and by assuming the tailward extension of the magnetopause to be cylindrical 
as was done e.g. by GALL et al. (1984). The result of this modification is shown in Fig. 10 for Deep River (geographic latitude: $46.10^{\circ}$, geographic longitude: $282.50^{\circ}$ ), again for December 7, 1982, 24:00 UT, and $K p \geqq 3+$. With the original TSYGANENKO-USMANOV (1982) model all the entry points of the particle trajectories for rigidities above $1.18 \mathrm{GV}$ were at $25 \mathrm{r}_{\mathrm{e}}$ in the tail region and not on the magnetopause. Figure 10 demonstrates that as far as the directions of approach are concerned, a spatial extension of the magnetospheric tail field leads to noticeable changes for rigidities below approximately $2 \mathrm{GV}$.

A further shortcoming of the TSYGANENKO-USMANOV (1982) model is its limited range in geomagnetic activity and the fact that asymmetric effects due to field-aligned currents are not taken into account. The results of our calculations indicate that the effects of perturbations in the magnetospheric magnetic field on cosmic rays may well be of the same order of magnitude as the effects of the quiescent external magnetic field with respect to the internal field. This clearly demonstrates the need for accurate models of the Earth's external magnetic field for all levels of geomagnetic activity.

It appears to be a wide-spread opinion that computational costs are probably one of the major reasons why in most calculations of cosmic ray particle trajectories in nearEarth space only the internal geomagnetic field is used. Our analysis demonstrates that this is not necessarily true, although it confirms that the calculation of cosmic ray trajectories is in itself a computer intensive problem. As an example, on a IBM 3090-28E (and performing all numerical operations in double precision) of the order of 20 minutes were needed to obtain the results shown in Fig. 9. The calculations utilizing just the Earth's internal field took about 9 minutes, whereas the calculations utilizing the combined models of the Earth's internal and external field required only about two minutes more.

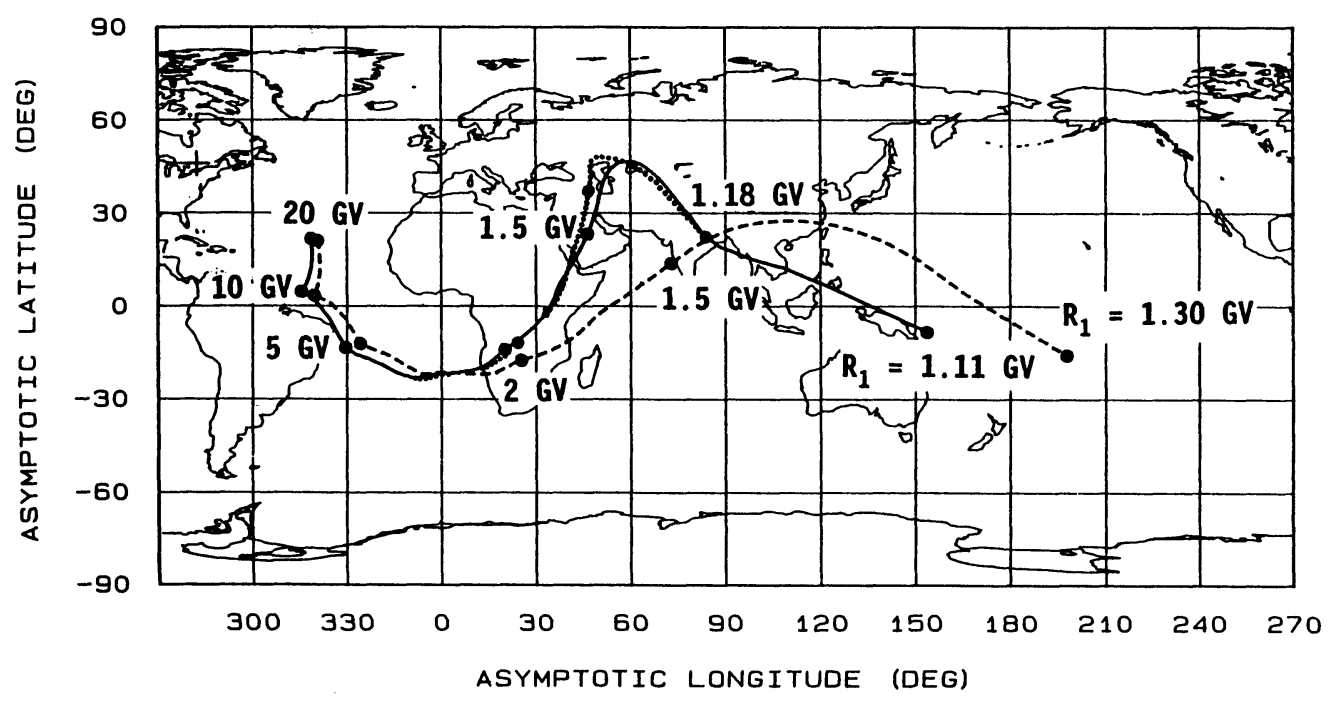

Fig. 10. Directions of approach (asymptotic directions) in geographic coordinates for cosmic ray particles arriving from the vertical direction at Deep River on December 7, 1982, 24:00 UT. Dotted line: with extended magnetospheric tail as described in text. Full line: without extended magnetospheric tail. Dashed line: IGRF 1980.0. 


\section{Summary and Conclusions}

It has been shown that any mathematical model of the main geomagnetic field is limited in its use for cosmic ray analyses, especially for the interpretation of cosmic ray observations made at high latitudes and/or during geomagnetic storms. For such studies models of the Earth's internal and external magnetic field have to be combined. As an example the combination of the IGRF 1980.0 field model with the TSYGANENKOUSMANOV (1982) magnetospheric model was discussed. It was shown that the task of combining the two models is primarily a problem of coordinate transformations where the transformation matrices depend on date and time. The evaluation of a suitable mathematical representation of the magnetopause needed for the cosmic ray trajectory calculations was also discussed. In addition, the consequences of combining internal and external magnetic field models on computer time were illustrated.

Finally we would like to emphasize that mathematical models which combine the Earth's internal and external magnetic field and which can be handled easily by the users are badly needed and a necessary prerequisite for many of today's cosmic ray analyses.

The authors are grateful to M. A. Shea and D. F. Smart for the trajectory-tracing program and the continuous interest in this work, and they thankfully acknowledge discussions with $\mathrm{H}$. Debrunner. This work was supported by the Swiss National Science Foundation, grant NF 2000-005285.

\section{REFERENCES}

DeBrunNer, H. and E. FlÖCKIgER, On the cutoff variations during the magnetic disturbances of 30.3.-3.4.1973 and 13.-18.9.1974, 15th Intl. Cosmic Ray Conf., Plovdiv, Bulgaria, Conf. Pap. 11, 151, 1977.

Duffet-Smith, P., Practical Astronomy with Your Calculator, 2nd ed., Cambridge University Press, 1981.

FAIRFIELD, D. H., On the accuracy of geomagnetic field models, EOS, Trans. Am. Geophys. Union, 69, 426, 1988.

FLÜCKIGER, E. O., Effects of magnetospheric currents on cosmic rays, in Invited Talks, 9th European Cosmic Ray Symposium, edited by K. Kudela and S. Pinter, p. 105, Slovak Academy of Sciences, Institute of Experimental Physics, Kosice, Czechoslovakia, 1984.

Flúckiger, E. O., D. F. SMART, and M. A. Shea, The effect of local perturbations of the geomagnetic field on cosmic ray cutoff rigidities at Jungfraujoch and Kiel, J. Geophys. Res., 88, 6961, 1983.

FlüCKIger, E. O., D. F. SMART, and M. A. SheA, A procedure for estimating the changes in cosmic ray cutoff rigidities and asymptotic directions at low and middle latitudes during periods of enhanced geomagnetic activity, J. Geophys. Res., 91, 7925, 1986.

Gall, R., A. Orozco, C. Marin, A. Hurtado, and G. Vidargas, Tables of Approach Directions and Points of Entry of Cosmic Rays for Higher Latitude Cosmic Ray Stations, Instituto de Geofisica, Universidad Nacional Autonoma de Mexico, 1984.

GiLL, S., A process for the step-by-step integration of differential equations in an automatic digital computing machine, Proc. Cambridge Phil. Soc., 47, 96, 1951.

IAGA Commission 2 Working Group 4, International Geomagnetic Reference Field 1965.0, J. Geophys. Res., 74, 4407, 1969.

IAGA Division 1 Working Group 1, IGRF 1980, EOS, Trans. Am. Geophys. Union, 62, 1169, 1981.

KoBEL, E., Bestimmung der Grenzsteifigkeiten und der Asymptotischen Richtungen der Kosmischen Strahlung für das solare Protonenereignis vom 7./8. Dezember 1982 unter Berücksichtigung der Einflüsse der gestörten Erdmagnetosphäre, Lizentiatsarbeit, Physikalisches Institut, Universität Bern, 1989.

Kudo, S., M. Wada, P. TAnskanen, and M. Kodama, Local time and cutoff rigidity dependences of storm time increase associated with geomagnetic storms, J. Geophys. Res., 92, 4719, 1987. 
Lemaitre, G. and M. S. Vallarta, On the allowance cone of cosmic radiation, Phys. Rev., 50, 493, 1936.

McCracken, K. G., The cosmic-ray flare effect. I. Some new methods of analysis, J. Geophys. Res., 67, 423, 1962.

McCracken, K. G., U. R. Rao, and M. A. Shea, The trajectories of cosmic rays in a high degree simulation of the geomagnetic field, MIT Tech. Report. No. 77, NYO-2670, 1962.

McCracken, K. G., U. R. Rao, B. C. Fowler, M. A. Shea, and D. F. Smart, Cosmic Rays, Annals of the IQSY, Vol. 1, edited by C. M. Minnis, Chapter 14, p. 198, The MIT Press, Cambridge, Massachusetts, 1968.

MEAD, G. D. and D. H. Fairfield, A quantitative magnetospheric model derived from spacecraft magnetometer data, J. Geophys. Res., 80, 523, 1975.

Olson, W. P., Coordinate Transformations Used in Magnetospheric Physics, MDAC Paper WD 1145, McDonnell Douglas Astronautics Company, 1970.

Pfitzer, K. A., W. P. Olson, and T. Mogstad, A time dependent, source driven magnetospheric field model, EOS, Trans. Am. Geophys. Union, 69, 426, 1988.

Shea, M. A. and D. F. Smart, Asymptotic directions and vertical cutoff rigidities for selected cosmic-ray stations as calculated using the International Geomagnetic Reference Field model appropriate for Epoch 1975.0, ERP No. 510, AFCRL-TR-75-0247, ADA015736, 1975.

Smart, D. F., M. A. Shea, and L. C. Gentile, Vertical cutoff rigidities calculated using the estimated 1985 geomagnetic field coefficients, 20th Intl. Cosmic Ray Conf., Conference Papers 4, 204, 1987.

Stern, D. P., Representation of magnetic fields in space, Rev. Geophys. Space Phys., 14, 199, 1976.

Störmer, C., The Polar Aurora, Oxford University Press, Fair Lawn, New Jersey, 1955.

Tsyganenko, N. A., A model of the cislunar magnetospheric field, Ann. Geophys., 32, 1, 1976.

Tsyganenko, N. A., A magnetospheric magnetic field model with a Warped Tail Current Sheet, Planet. Space Sci., 37, 5, 1989.

TSyganenko, N. A. and A. V. Usmanov, Determination of the magnetospheric current system parameters and development of experimental geomagnetic field models based on data from IMP and HEOS satellites, Planet. Space Sci., 30, 985, 1982.

WALKER, R. J., Quantitative modeling of planetary magnetospheric magnetic fields, in Quantitative Modeling of Magnetospheric Processes, edited by W. P. Olson, p. 9, Geophysical Monograph 21, American Geophysical Union, Washington D.C., 1979.

Walker, R. J., Modeling planetary magnetospheres, Rev. Geophys. Space Phys., 21, 495, 1983.

Wilken, B., D. N. Baker, P. R. Higbie, T. A. Fritz, W. P. Olson, and K. A. Pfitzer, Magnetospheric configuration and energetic particle effects associated with a SSC: A case study of the CDAW 6 event on March 22, 1979, J. Geophys. Res., 91, 1459, 1986. 